\title{
Cognitive impairment in depression and its (non-)response to antidepressant treatment
}

D Chamith Halahakoon, Jonathan P Roiser

Institute of Cognitive Neuroscience, University College London, London, UK

Correspondence to:

Dr Jonathan P Roiser

UCL Institute of Cognitive Neuroscience 17 Queen Square

London

United Kingdom

WC1N 3AZ

Email: j.roiser@ucl.ac.uk

Citation: Shilyansky C, Williams LM, Gyurak A, et al. Effect of antidepressant treatment on cognitive impairments associated with depression: a randomised longitudinal study. Lancet Psychiatry 2016;3:425-35.

\section{Commentary:}

\section{What is already known on this topic}

Cognitive impairment is a reliable feature of major depressive disorder. Depressed individuals perform more poorly than never-depressed healthy volunteers across a range of neuropsychological tests. Cognitive impairments predict poor response to antidepressant drugs, persist after successful treatment [1] and are associated with poor psychosocial functioning.[2] Remediation of cognitive function in depression, for example by using cognitive training strategies [3] or cognitive-enhancing drugs such as modafinil,[4] have been shown to boost recovery from depression. The current study aimed to test whether standard antidepressant treatment improves cognitive function in depression.

\section{Methods of the study}

This was a longitudinal, randomised, open-label trial assessing the effects of three antidepressant drugs on cognitive function in depressed individuals. There were three treatment arms (one for each drug) as well as an untreated healthy control group. Cognitive performance in depressed patients was assessed at baseline and following 8 weeks of treatment; testing was completed in the control group at the same intervals. The raters who carried out clinical assessments were blind to treatment arm and cognitive performance 
throughout the trial. Enrolment of depressed individuals and healthy controls took place between 08 December 2008 and 30 September 2011 at 17 sites across the USA, New Zealand, South Africa, Australia and the Netherlands. Exclusion criteria for depressed individuals included a baseline score below 17 on the Hamilton Rating Scale for Depression (HRSD-17), a history of failure to respond or contraindications to any protocol drug or comorbidities that might interfere with the completion of assessments. A total of 1008 depressed individuals and 336 never-depressed healthy controls were enrolled. Depressed participants, all of whom were unmedicated for at least the week preceding the start of the trial, were assigned to one of three oral antidepressants (escitalopram, sertraline or venlafaxine-XR) using a blocked randomisation procedure (with a block size of 12). At weeks 0 (pre-treatment) and 8 (post-treatment), trained study personnel assessed depressive symptoms using the HRSD-17 and Quick Inventory of Depression Symptomatology (QIDSSR16); average baseline scores indicated a moderate-to-severe degree of symptoms. Cognitive function was measured using the computerised 'IntegNeuro' cognitive battery which comprised the following tests: attention (1-back continuous performance test); response inhibition (go-no go task); verbal memory (verbal interference task); executive function (Austin maze); cognitive flexibility (Stroop task); working memory (forward digit span); decision speed (choice reaction time); information processing (switching of attention between numbers and letters in a trails $A$ and $B$ task); and motor coordination (finger tapping). Scores of $\leq 7$ on the HDRS-17 and $\leq 5$ on the QIDS-SR16 at week 8 were used to define 'remitter' and 'non-remitter' groups. The analyses included 712 patients and 316 controls who completed the study.

\section{What this paper adds}

- Owing to the large sample size, this study provides the clearest evidence to-date for the existence of cognitive impairment in depression and its resistance to treatment.

- Baseline assessments confirmed significant cognitive impairment in depression on all tests (Cohen's d effect sizes for healthy controls vs patients at week 0 ranged from 0.20 to 0.46 ) except for the forwards digit span ( $d=0.02$ ).

- Cognitive impairment in depressed individuals, relative to healthy volunteers, was not significantly affected by either antidepressant administration or treatment success, except for the executive function, which was the only test in which performance improved over the 8-week study period to a significantly greater extent in patients than controls.

- There were no significant differences between the three treatment arms in postintervention cognitive functioning, suggesting that the protocol drugs were similarly ineffective, at the doses used, in treating cognitive impairment in depression.

\section{Limitations}

- A depressed group who were administered a placebo would have strengthened the design. In particular, this might have clarified the extent to which practice effects contributed to the apparent improvement on the Austin maze task (performance on which reflects a variety of cognitive processes) observed following antidepressant treatment; this finding conflicts with previous studies that used a placebo-controlled design.[5] 
- There was a low completion rate in the depressed group compared to the control group, $71 \%(712 / 1008)$ and $94 \%$ (316/336), respectively, with no significant differences between treatment arms. Depressed participants that dropped out performed significantly worse on a test of attention at baseline than those who completed the study. This may have produced a bias in the analyses, which only included those who completed all assessments. This could be addressed by employing statistical techniques to correct for missing data, such as multiple imputation.

\section{What next in research}

- Large-scale trials assessing the ability of cognitive enhancing drugs or training techniques to augment existing treatments for depression will be necessary to test whether these strategies are effective, as suggested by preliminary work.[3,4]

- The study of cognitive impairment in depression as an indicator of response-potential to different treatment modalities, including psychological therapies, may assist clinicians in better tailoring treatment plans to individual patients.

- Trials assessing the effectiveness of other currently available antidepressants (and treatment modalities) at remediating cognitive impairment, as well as the development of novel treatment strategies specifically targeting cognitive impairment in depression, may be useful in helping to address this aspect of the condition.

\section{Do these results change your practices and why?}

No. As the trial drugs were similarly ineffective at improving cognitive impairment in depression at the doses used, these results are unlikely to cause an immediate change in standard treatment for depression. However, they may encourage health authorities to consider the importance of cognitive impairment in depression when formulating treatment guidelines.

Funding: DCH and JPR are funded by the Wellcome Trust.

Competing interests: JPR is a consultant for Cambridge Cognition.

\section{References}

1. Rock PL, Roiser JP, Riedel WJ, et al. Cognitive impairment in depression: a systematic review and meta-analysis. Psychol Med 2014;44:2029-40.

2. Jaeger J, Berns $S$, Uzelac $S$, et al. Neurocognitive deficits and disability in major depressive disorder. Psychiatry Res 2006;145:39-48.

3. Porter R, Douglas K, Jordan J, et al. Psychological treatments for cognitive dysfunction in major depressive disorder: current evidence and perspectives. CNS Neurol Disor Drug Targets 2014;13:1677-92. 
4. Abolfazli R, Hosseini M, Ghanizadeh A, et al. Double-blind randomized parallel-group clinical trial of efficacy of the combination fluoxetine plus modafinil versus fluoxetine plus placebo in the treatment of major depression. Depress Anxiety 2011;28:297-302.

5. Rosenblat JD, Kakar R, Mclntyre RS, et al. The cognitive effects of antidepressants in major depressive disorder: a systematic review and meta-analysis of randomized clinical trials. Int J Neuropsychopharmacol 2016;19:pyv082. 